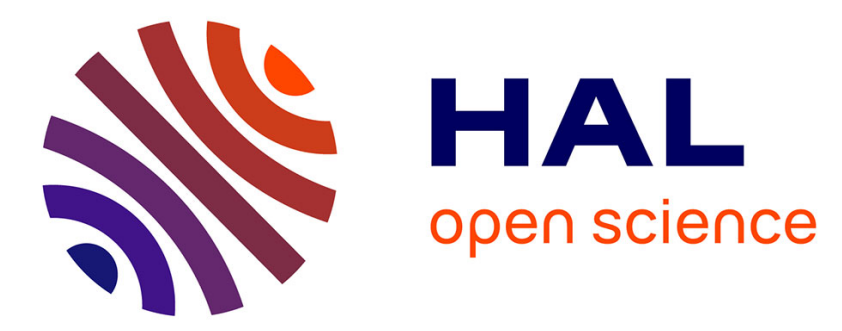

\title{
LMTO-ASA method for the electronic structure of dilute alloys
}

\author{
C. Koenig, E. Daniel
}

\section{To cite this version:}

C. Koenig, E. Daniel. LMTO-ASA method for the electronic structure of dilute alloys. Journal de Physique Lettres, 1981, 42 (9), pp.193-195. 10.1051/jphyslet:01981004209019300 . jpa-00231907

\section{HAL Id: jpa-00231907 https://hal.science/jpa-00231907}

Submitted on 1 Jan 1981

HAL is a multi-disciplinary open access archive for the deposit and dissemination of scientific research documents, whether they are published or not. The documents may come from teaching and research institutions in France or abroad, or from public or private research centers.
L'archive ouverte pluridisciplinaire HAL, est destinée au dépôt et à la diffusion de documents scientifiques de niveau recherche, publiés ou non, émanant des établissements d'enseignement et de recherche français ou étrangers, des laboratoires publics ou privés. 


\title{
LMTO-ASA method for the electronic structure of dilute alloys
}

\author{
C. Koenig and E. Daniel \\ Laboratoire de Magnétisme et de Structure Electronique des Solides (*), \\ Université Louis-Pasteur, 4, rue Blaise-Pascal, 67070 Strasbourg Cedex, France \\ (Reçu le 16 février 1981, accepté le 9 mars 1981)
}

\begin{abstract}
Résumé. - Partant de la fonction de Green pour une impureté dans un métal, et effectuant le passage à la limite convenable, nous établissons les formules équivalentes dans l'approximation LMTO-ASA.
\end{abstract} Abstract. - Starting from the Green function formalism of the impurity problem, we derive the corresponding
LMTO-ASA formulas by taking the proper limit.

The Green function method (KKR [1]), which has been used for numerous self-consistent band structure calculations of pure metals, has been formally extended to the problem of dilute impurities by several authors, among whom Beeby [2] and Harris [3]. It was recently applied by Zeller and Dederichs [4], then by Podloucky et al. [5] (hereafter referred as PZD) to the self-consistent calculation of the electronic structure of substitutional impurities in $\mathrm{Cu}$ and Ag. Nevertheless, it is rather cumbersome to bring it numerically into play. We have recently shown [6] that the linear muffin-tin orbitals method (LMTO-ASA [7]) can be satisfactorily applied to the computation of the electronic density of states on the impurity site. This method, which treats as in KKR the matrix and the impurity on the same footing, is very efficient in saving computational time and its results are in very good agreement with those of the KKR method. Moreover, our numerical results on s-p impurities in ferromagnetic iron show that, if the Friedel's sum rule is satisfied, the impurity cell is generally not neutral, so that the induced effect on its nearest neighbours must be estimated.

In this paper, starting from the formulas given by Podloucky et al. [5] and keeping their notations, we derive the corresponding formulas for the Green function of the alloy within the LMTO approximation.
With the usual conventional notations [1], the KKR secular equation is written as :

$$
\left|B_{L L^{\prime}}^{k}(\varkappa)+\varkappa \operatorname{cotg} \eta_{l} \delta_{L L^{\prime}}\right|=0 \quad L=(l, m) .
$$

Let $A$ be the diagonal matrix defined by :

$$
A_{L L^{\prime}}(\varkappa)=A_{l}=\frac{(2 l-1) ! !}{\sqrt{2 S}(\varkappa S)^{l}} \delta_{L L^{\prime}}
$$

where $S$ is the radius of the muffin-tin sphere, and the operators $S$ and $P$ by :

$$
\begin{gathered}
\delta_{L L^{\prime}}^{k}=\operatorname{Lim}_{\chi \rightarrow 0} A_{l}^{-1} B_{L L^{\prime}}^{k}(\chi) A_{l}^{-1} \\
P_{l}(E)=\operatorname{Lim}_{x \rightarrow 0} A_{l}^{-1}\left(-\varkappa \operatorname{cotg} \eta_{l}\right) A_{l}^{-1}= \\
=\operatorname{Lim}_{x \rightarrow 0} A_{l}^{-1} t_{L}^{-1}(E) A_{l}^{-1}
\end{gathered}
$$

Andersen [7] has shown that in the limit where $\varkappa^{2}$ goes to zero, (1) is equivalent to :

$$
\left|\delta_{L L^{\prime}}^{k}-P_{l}(E) \delta_{L L^{\prime}}\right|=0 .
$$

We shall take the same limit $x \rightarrow 0$ in the expression (6) of the Green function given by Podloucky et al. ([5], formula PZD (2)) :

$$
\begin{aligned}
G\left(\mathbf{R}^{m}+\mathbf{r}, \mathbf{R}^{m^{\prime}}+\mathbf{r}^{\prime}\right)=\sum_{L} \chi R_{l}^{m}\left(r_{<}, E\right) Y_{L}(\hat{r}) H_{l}^{m}\left(r_{>}, E\right) Y_{L^{\prime}}\left(\hat{r}^{\prime}\right) \delta_{m m^{\prime}}+ & \sum_{L L^{\prime}} R_{l}^{m}(r, E) Y_{L}(\hat{r}) \times \\
& \times G_{L L^{\prime}}^{m m^{\prime}}(E) R_{l^{\prime}}^{m^{\prime}}\left(r^{\prime}, E\right) Y_{L^{\prime}}\left(\hat{r}^{\prime}\right) .
\end{aligned}
$$

$\left(^{*}\right)$ L.A. au C.N.R.S. no 306. 
The first term corresponds to the scattering by an isolated potential on site $m$ in free space. The second term is given by the Dyson equation :

$$
\begin{aligned}
& \tilde{G}_{L L^{\prime}}^{m m^{\prime}}(E)=\tilde{G}_{L L^{\prime}}^{0\left(m-m^{\prime}\right)}(E)+ \\
& \quad+\sum_{n L^{\prime \prime}} \widetilde{G}_{L L^{\prime \prime}}^{0(m-n)}(E) \Delta t_{l^{\prime \prime}}^{n}(E) \widetilde{G}_{L^{\prime \prime} L^{\prime}}^{n m^{\prime}}(E)
\end{aligned}
$$

where

$$
\widetilde{G}_{L L^{\prime}}^{m m^{\prime}}=\mathrm{e}^{-i \eta_{l}^{m}} G_{L L^{\prime}}^{m m^{\prime}} \mathrm{e}^{-i \eta_{l^{\prime}}^{m \prime^{\prime}}}
$$

and the zero subscript refers to the pure matrix. The product $A_{l} R_{l}^{m}(r, E)$ has a finite limit as $x \rightarrow 0$ (see [7], formulas $(4.22 b),(2.6),(2.10)$ and (2.17)) :

$$
\begin{aligned}
\operatorname{Lim}_{x \rightarrow 0}[ & \left.A_{l} R_{l}^{m}(r, E)\right]=\left(P_{l}^{m}(E)\right)^{-1} \times \\
& \times \frac{2 l+1}{D_{l}^{m}(E)-l} \sqrt{\frac{2}{S}} \frac{\Phi_{l}^{m}\left(r, D_{l}^{m}(E)\right)}{\Phi_{l}^{m}\left(S, D_{l}^{m}(E)\right)}=\left(P_{l}^{m}(E)\right)^{-1} \\
& \times \sqrt{\dot{P}_{l}^{m}(E)} \Phi_{l}^{m \mathrm{~N}}(r, E)
\end{aligned}
$$

where $\Phi_{l}^{m}\left(r, D_{l}(E)\right)$ is the radial solution of the Schroedinger equation in the sphere $m$, with a logarithmic derivative

$$
D_{l}^{m}(E)=\left(r \frac{\Phi_{l}^{\prime m}(r)}{\Phi_{l}^{m}(r)}\right)_{r-s}
$$

on the sphere boundary. $\Phi_{l}^{m \mathrm{~N}}(r, E)$ is the solution normalized to 1 in the same sphere. The connection between $P_{l}^{m}(E)$ and $D_{l}^{m}(E)$ is given by :

$$
P_{l}^{m}(E)=2(2 l+1) \frac{D_{l}^{m}(E)+l+1}{D_{l}^{m}(E)-l} .
$$

The product $\left[A_{l}^{-1} G_{L L^{\prime}}^{m m^{\prime}}(E) A_{l}^{-1}\right]$ can then be factorized in the second term of equation (6) and has also a finite limit :

$$
\Gamma_{L L^{\prime}}^{m m^{\prime}}(E)=\operatorname{Lim}_{x \rightarrow 0} A_{l}^{-1} G_{L L^{\prime}}^{m m^{\prime}}(E) A_{l^{\prime}}^{-1}
$$

In the limit $x \rightarrow 0$, the phase shifts $\eta_{l} \rightarrow 0$ so that the Dyson equation (7) becomes :

$$
\begin{aligned}
\Gamma_{L L^{\prime}}^{m m^{\prime}}(E)= & \Gamma_{L L^{\prime}}^{0\left(m-m^{\prime}\right)}(E)+ \\
& +\sum_{n L^{\prime \prime}} \Gamma_{L L^{\prime \prime}}^{0(m-n)} \Delta\left(P_{l^{\prime \prime}}^{n}(E)^{-1}\right) \Gamma_{L^{\prime \prime} L^{\prime}}^{n m^{\prime}}(E) .
\end{aligned}
$$

We first determine the intersite $\left(m \neq m^{\prime}\right)$ elements of the host Green function $\Gamma^{0}$. The Bloch wave function on a site $m$ is :

$$
\begin{aligned}
\Psi_{\mathbf{k} j}^{0}\left(\mathbf{R}^{m}+\mathbf{r}\right) & =\mathrm{e}^{i \mathbf{k} \cdot \mathbf{R}^{m}} \sum_{L} i^{l}\left[A_{l}^{-1} \phi_{L}(\mathbf{k}, j)\right] \times \\
& \times\left[A_{l} R_{l}^{0}(r, E)\right] Y_{L}(\hat{r}) . \quad(\mathrm{PZD}
\end{aligned}
$$

Let us define :

$$
F_{L}(\mathbf{k}, j)=\operatorname{Lim}_{x \rightarrow 0}\left[A_{l}^{-1} \phi_{L}(\mathbf{k}, j)\right] .
$$

This quantity can be numerically obtained from (8) and the limit of (12) which gives the Bloch wave function in the matrix within the LMTO scheme.

For $m \neq m^{\prime}$, the imaginary part of $\Gamma^{0}$ is given by :

$$
\begin{array}{r}
\operatorname{Im} \Gamma_{L L}^{0\left(m-m^{\prime}\right)}(E)=-\pi \sum_{j} \int_{\mathrm{BZ}} \mathrm{d}^{3} k \delta\left(E-E_{j k}\right) \times \\
\times F_{L}(\mathbf{k}, j) F_{L^{\prime}}^{*}(\mathbf{k}, j) i^{l-l^{\prime}} \mathrm{e}^{i \mathbf{k} .\left(\mathbf{R}^{m}-\mathbf{R}^{m^{\prime}}\right)} .
\end{array}
$$

The real part is obtained by a Kramers-Krönig transformation (PZD 15) :

$$
\begin{aligned}
\beta_{l l^{\prime}}(E) \operatorname{Re} \Gamma_{L L^{\prime}}^{0\left(m-m^{\prime}\right)}(E) & =\frac{1}{\pi} \int_{-\infty}^{\infty} \mathrm{d} E^{\prime} \mathcal{T} \cdot\left(\frac{1}{E-E^{\prime}}\right) \times \\
\times & \beta_{l l^{\prime}}\left(E^{\prime}\right) \operatorname{Im} \Gamma_{L L^{\prime}}^{0\left(m-m^{\prime}\right)}(E)
\end{aligned}
$$

with

$$
\begin{aligned}
& \beta_{l l^{\prime}}(E)=\operatorname{Lim}_{x \rightarrow 0}\left[A_{l} \alpha_{l l^{\prime}}(E) A_{l^{\prime}}\right]= \\
& =\operatorname{Lim}_{x \rightarrow 0} \int_{0}^{s} r^{2} \mathrm{~d} r\left[A_{l} R_{l}^{0}(r, E)\right]\left[A_{l^{\prime}} R_{l^{\prime}}^{0}(r, E)\right] .
\end{aligned}
$$

$\beta_{l l^{\prime}}(E)$ can be obtained numerically from (8), so that the Green function $\Gamma_{L L^{\prime}}^{0\left(m-m^{\prime}\right)}(E)$ is completely determined.

In the case $m=m^{\prime}, \Gamma^{0}$ can be related to the operator $S(E)$ defined by Harris [3] by identifying term by term the expansions of the matrix Green function (the site index is now suppressed) :

$$
\begin{aligned}
& \left(G-G^{\mathbf{S}}\right)\left(\mathbf{r}, \mathbf{r}^{\prime}, E\right)= \\
& \quad=\sum_{L L^{\prime}} \Delta_{L}(\mathbf{r}, E)(S(E)-t(E))_{L L^{\prime}} \Delta_{L^{\prime}}(\mathbf{r}, E)
\end{aligned}
$$

([3], (3.7), (3.11)) where

$$
S(E)=\frac{1}{\tau} \int_{\mathrm{ZB}} \mathrm{d}^{3} k \frac{1}{t^{-1}(E)-\tau G^{0}(\mathbf{k}, E)}
$$

and where the wave function $\Delta_{L}(\mathbf{r}, E)$ is related to $R_{L}(\mathbf{r}, E)$ through :

$$
\Delta_{L}(\mathbf{r}, E)=\mathrm{e}^{i \eta_{l}} t_{l}^{-1}(E) R_{L}(\mathbf{r}, E) .
$$

It is easily seen that, on a matrix site, one has, in the notations of PZD :

$\widetilde{G}^{0}=t^{-1} S t^{-1}-t^{-1}$ and $\Gamma^{0}=P \Sigma P-P$,

where $\Sigma=\operatorname{Lim}_{x \rightarrow 0}$ ASA.

We have shown elsewhere [6] how to calculate $\Sigma_{L}=\sigma_{L}+i \zeta_{L}$ from the density of states $n_{L}(E)$ of the matrix, following the remarks of Harris [3]. The only subtle point arises from the fact that, at the limit $x \rightarrow 0$, the Green function $G^{\mathrm{S}}$ gives always a bound state for each value of $l$, at an energy $E_{l}^{*}$ satisfying the condition $D_{l}\left(E_{l}^{*}\right)=-l-1$. 


$$
\left.\begin{array}{l}
\sigma_{L}(E)=\dot{P}_{l}(E)^{-1} \mathfrak{T} \cdot\left[\int_{-\infty}^{\infty} \frac{n_{L}\left(E^{\prime}\right) \mathrm{d} E^{\prime}}{E-E^{\prime}}-\frac{1}{E-E_{l}^{*}}\right]+P_{l}(E)^{-1} \\
\zeta_{L}(E)=-\frac{1}{\pi} \dot{P}_{l}(E)^{-1} n_{L}(E) .
\end{array}\right\}
$$

The Green function $\Gamma_{L}^{0}=x_{L}+i y_{L}$ on a matrix site is then given by :

$$
\left.\begin{array}{l}
x_{L}(E)=P_{l}(E) \sigma_{L}(E) P_{l}(E)-P_{l}(E) \\
y_{L}(E)=P_{l}(E) \zeta_{L}(E) P_{l}(E) .
\end{array}\right\}
$$

In particular, one obtains from the Dyson equation (11) the same formula for the density of electronic charge on the impurity site as the one that we obtained previously ([6], formula 11) :

$$
\rho_{L}^{\prime}(\mathbf{r}, E)=-\frac{1}{\pi}\left[\Phi_{L}^{\prime N}(\mathbf{r}, E)^{2}\right] \dot{P}_{l}^{\prime}(E) \operatorname{Im} \frac{1}{P_{l}^{\prime}(E)-P_{l}(E)+\Sigma_{l}^{-1}(E)}
$$

where all the primed quantities refer to the impurity. On the impurity site, the equation (11) is immediately solved :

$$
\Gamma=\frac{\Gamma^{0}}{1-\left(\frac{1}{P^{\prime}}-\frac{1}{P}\right) \Gamma^{0}} .
$$

In the limit $x \rightarrow 0$ and taking (8) into account, the density of electronic charge is :

$\rho_{L}^{\prime}(\mathbf{r}, E)=-\frac{1}{\pi} \operatorname{Lim}_{x \rightarrow 0}\left[A_{l} R_{L}^{\prime}(\mathbf{r}, E)\right]\left[A_{l}^{-1} G_{L L} A_{l}^{-1}\right]\left[A_{l} R_{L}^{\prime}(\mathbf{r}, E)\right]=-\frac{1}{\pi}\left[\Phi_{L}^{\prime N}(\mathbf{r}, E)\right]^{2} \dot{P}_{l}^{\prime}(E) \operatorname{Im} \frac{\Gamma_{l}}{P_{l}^{\prime 2}(E)}$.

From $(19 b)$ and (21), one obtains directly :

$$
\operatorname{Im} \Gamma_{l}=\operatorname{Im} \frac{P_{l}^{\prime 2}}{P_{l}^{\prime}-P_{l}+\Sigma_{l}^{-1}}
$$

so that (22) and (20) are identical.

As in the case of pure metals, the wave functions on the matrix or impurity sites are not computed at each energy, but are linearly expanded around a given energy $E_{v}$, chosen in the vicinity of the centre of gravity of the occupied states :

$$
\Phi_{L}^{m}(\mathbf{r}, E)=\Phi_{L}^{m}\left(\mathbf{r}, E_{v}\right)+\left(E-E_{v}\right) \dot{\Phi}_{L}^{m}\left(\mathbf{r}, E_{v}\right) .
$$

The atomic sphere approximation (ASA) consists in replacing in the LMTO formulas developed above the muffin-tin sphere by the Wigner-Seitz sphere. It is the simplest way of getting rid of the interstitial region, poorly treated in the muffin-tin approximation. This is as well justified as for pure metals [7].

In this paper, we derived the formulas giving the intersite Green function in a dilute alloy within the LMTO-ASA approximation. This method, which has already given good numerical results for the density of states on the impurity site, should allow to go beyond the central site approximation and to estimate the effect of the perturbation induced in the matrix by the impurity, with the same numerical advantages with respect to the Green function method as in the case of pure metals.

\section{References}

[1] Kohn, W., Rostoker, N., Phys. Rev. 94 (1954) 1111. Ham, F. S., Segall, B., Phys. Rev. 124 (1961) 1784.

[2] Beeby, J. L., Proc. R. Soc. A 302 (1967) 113.

[3] Harris, R., J. Phys. C 3 (1970) 172.

[4] Zeller, R., Dederichs, P. H., Phys. Rev. Lett. 42 (1979) 1913.
[5] Podloucky, R., Zeller, R., Dederichs, P. H., Phys. Rev. B 22 (1980) 5777.

[6] Koenig, C., Léonard, P., Daniel, E., to be published in $J$. Physique 42 (1981).

[7] Andersen, O. K., Phys. Rev. B 12 (1975) 3060. 